\title{
A generalization of Mortici lemma
}

\author{
VASILE BERINDE
}

\section{ABSTRACT.}

The aim of this note is to obtain a generalization of a very simple, elegant but powerful convergence lemma introduced by Mortici [Mortici, C., Best estimates of the generalized Stirling formula, Appl. Math. Comp., 215 (2010), No. 11, 4044-4048; Mortici, C., Product approximations via asymptotic integration, Amer. Math. Monthly, 117 (2010), No. 5, 434-441; Mortici, C., An ultimate extremely accurate formula for approximation of the factorial function, Arch. Math. (Basel), 93 (2009), No. 1, 37-45; Mortici, C., Complete monotonic functions associated with gamma function and applications, Carpathian J. Math., 25 (2009), No. 2, 186-191] and exploited by him and other authors in an impressive number of recent and very recent papers devoted to constructing asymptotic expansions, accelerating famous sequences in mathematics, developing approximation formulas for factorials that improve various classical results etc.

We illustrate the new result by some important particular cases and also indicate a way for using it in similar contexts.

TECHNiCAl University OF Cluj-NAPOCA

NORTH UniVERSiTy CENTER OF BAIA MARE

DEPARTMENT OF MATHEMATICS AND COMPUTER SCIENCE

Victoriei 76, 430122 BAiA MARE, ROMANiA

E-mail address: vberinde@ubm.ro 